\title{
Spinocerebellar ataxia in the Italian Spinone dog is associated with an intronic GAA repeat expansion in ITPRI
}

\author{
Oliver P. Forman - Luisa De Risio • \\ Kaspar Matiasek · Simon Platt • Cathryn Mellersh
}

Received: 22 July 2014/ Accepted: 5 September 2014/Published online: 30 October 2014

(C) The Author(s) 2014. This article is published with open access at Springerlink.com

\begin{abstract}
Spinocerebellar ataxia in the Italian Spinone dog breed is characterised by a progressive gait abnormality that manifests from approximately 4 months of age. The disorder shows an autosomal recessive mode of inheritance, and affected individuals are usually euthanized by one year of age on welfare grounds due to an inability to ambulate. Using a homozygosity mapping technique with six cases and six controls, we mapped the disease locus to chromosome 20 of the canine genome. Linkage analysis across an extended pedigree confirmed the association, with microsatellite C20.374 achieving a maximal LOD score of 4.41. All five genes within the disease-associated interval were exon resequenced, although no exonic candidate mutations were identified. A targeted resequencing approach was therefore adopted to sequence the entire disease-associated interval. Analysis of the sequencing data
\end{abstract}

Electronic supplementary material The online version of this article (doi:10.1007/s00335-014-9547-6) contains supplementary material, which is available to authorized users.

O. P. Forman $(\bowtie) \cdot$ C. Mellersh

Kennel Club Genetics Centre, Animal Health Trust, Kentford,

Newmarket, Suffolk CB8 7UU, UK

e-mail: oliver.forman@aht.org.uk

L. De Risio

Neurology Department, Animal Health Trust, Kentford,

Newmarket, Suffolk CB8 7UU, UK

K. Matiasek

Section of Clinical \& Comparative Neuropathology, Institute of

Veterinary Pathology, Ludwig Maximilians University,

Veterinarstr 13, 80539 Munich, Germany

\section{S. Platt}

Department of Small Animal Medicine and Surgery, The

University of Georgia College of Veterinary Medicine,

501 D.W. Brooks Drive, Athens, GA 30602, USA revealed a GAA repeat expansion in intron 35 of ITPRI, which was homozygous in all cases and heterozygous in obligate carriers. Partial impairment of cerebellar ITPRI expression in affected dogs was demonstrated by immunohistochemistry. Given the association of ITPRI mutations with spinocerebellar ataxia (SCA) type 15 (also designated SCA16) in humans and that an intronic GAA repeat expansion has been shown to cause Friedreich ataxia, the repeat expansion is an excellent candidate for the cause of spinocerebellar ataxia in the Italian Spinone. This finding represents the first naturally occurring pathogenic intronic GAA repeat expansion in a non-human species and a novel mechanism for ITPRI associated spinocerebellar ataxia.

\section{Introduction}

Spinocerebellar ataxia in the Italian Spinone (SCAIS) is a progressive neurodegenerative disease characterised by hypermetria, particularly in the pelvic limbs, truncal ataxia and impaired balance. Clinical signs start to appear at four months of age and progress to a degree of dysfunction which leads to euthanasia of affected dogs at one year of age on average. The disease shows an inheritance consistent with an autosomal recessive mode. SCAIS was acknowledged in the veterinary literature as a brief clinical communication in 1996 (Wheeler and Rusbridge 1996), and has been reported anecdotally in a number of countries including Italy, UK, USA and Denmark. Cases are rare, with no new cases reported since the launch of a linkage based DNA test at the Animal Health Trust in 2008, enabling breeders to identify heterozygous carriers in their breeding lines. 
In humans, clinically distinct types of spinocerebellar ataxias have been well characterised and numerically catalogued into autosomal recessive (SCAR1-12) and autosomal dominant (ADCA or SCA1-36) forms. For many of these forms disease-associated genes have been identified, allowing candidate gene studies to be undertaken in other species, an approach regularly adopted when studying canine disorders, and recently successfully used to identify the cause of a neonatal cerebellar ataxia in the Beagle dog (Forman et al. 2012). A range of mutation types have been associated with SCA in humans, including genomic deletions, duplications, point mutations and both intronic and exonic repeat expansions. Polyglutamine expansions are associated with several SCA types in humans including SCA1, 2, 3, 6, 7 and 17 (Higgins et al. 1996; Koide et al. 1999; Lindblad et al. 1996; Orr et al. 1993; Riess et al. 1997; Sanpei et al. 1996). Intronic repeat expansions account for SCA10 (Matsuura et al. 2000) and Friedreich ataxia (Campuzano et al. 1996), and $5^{\prime}$ and $3^{\prime}$ UTR repeat expansions are associated with SCA8 (Koob et al. 1999) and SCA12 (Holmes et al. 1999), respectively. The variety of mutation type is a key consideration when studying the molecular biology of spinocerebellar ataxia to ensure that causal mutations are not overlooked or misidentified.

In this investigation, we used a homozygosity mapping approach using six SCAIS cases and six controls to map the disease-associated locus. Results were followed up by exon resequencing of interval genes and subsequently targeted resequencing using a massively parallel sequencing technique in an attempt to elucidate the causal mutation. After identification of the candidate gene mutation, the expression of the gene product and its potential involvement with neurodegeneration was evaluated in serial sections of the cerebellum of three clinically and genetically confirmed SCA cases.

In this manuscript, we describe the identification of a novel intronic GAA triplet repeat expansion in the inositol 1,4,5-trisphosphate receptor, type 1 (ITPRI) gene. This represents the first naturally occurring pathogenic repeat expansion of its type in a non-human species and a novel mechanism for ITPRI-associated spinocerebellar ataxia.

\section{Materials and methods}

Sample collection

All DNA samples were collected from Italian Spinoni (IS) in the general pet dog population. We collected residual blood samples that were drawn as part of a veterinary procedure or buccal swab samples that were collected by owners or by veterinarians. DNA was collected from historic cases in the form of formalin fixed paraffin embedded
(FFPE) cerebellum tissue. The clinical diagnosis of SCA was based on neurological examination by two board certified neurologists (LDR and SP) in four dogs and on revision of video footage and medical records in two other dogs.

Homozygosity mapping and linkage analysis

Homozygosity mapping was performed using DNA from six SCAIS cases and six obligate carriers. A set of 325 genome-wide microsatellites was genotyped, of which 284 had a genotyping frequency of $>90 \%$, and 260 were informative with a minor allele frequency $>5 \%$. PCRs were performed in multiplex reactions of three to ten markers using fluorescently labelled forward primers for detection of products by capillary electrophoresis on ABI3100 genetic analysers. Genotypes were scored using Genemapper v4.0. Results were analysed initially by calculating the ratio of major allele homozygotes in the case versus control groups and also by visual inspection for a pattern suggestive of linkage. Positive results were followed up by performing linkage analysis. An extended IS pedigree was drawn using the Cyrillic software package and data exported for two-point linkage analysis using MLINK software.

\section{Sequencing}

Exon resequencing and sequencing of an expanded GAA repeat allele were performed using a standard Sanger sequencing methodology, using Bigdye v3.1 chemistry. Sequencing products were separated on ABI3100 genetic analysers and data analysed using the Staden Gap4 software package.

Libraries for targeted resequencing of the disease-associated region were prepared using a custom SureSelect XT target enrichment kit (Agilent). Capture probes (baits) were design using the online tool e-array (https://earray.chem. agilent.com/earray/). Final libraries were quantified using the Kapa library quantification kit, and sequencing was performed at the Wellcome Trust Centre for Human Genetics, University of Oxford, on an Illumina HiSeq 2000. A $20 \mathrm{~Gb}$ dataset of $51 \mathrm{bp}$ paired-end reads was produced. Reads were aligned to the CanFam 2 genome sequence using BWA (Li and Durbin 2009), and SNP and INDEL calls made using GATK (McKenna et al. 2010). Read alignments were visualised using the Integrative Genome Viewer (IGV) (Thorvaldsdottir et al. 2013).

\section{Repeat expansion PCR}

Long range PCR was performed to amplify GAA repeat alleles. Reactions consisted of $2.5 \mu \mathrm{l} 10 \times$ Qiagen LR 
reaction buffer, $1.25 \mu \mathrm{l}$ dNTPs (10 mM each), $0.5 \mu \mathrm{M}$ primers (forward: GGTGAGGAGCATGTTCTGGT; reverse: TGTCTCAGCGGTTGAATGTC), $2 \mu \mathrm{l}$ Qiagen LongRange PCR Enzyme Mix and ultrapure water to a volume of $25 \mu$ l. Cycling parameters were $93{ }^{\circ} \mathrm{C}$ for $3 \mathrm{~min}$, followed by 35 cycles of $93{ }^{\circ} \mathrm{C}$ for $15 \mathrm{~s}, 62{ }^{\circ} \mathrm{C}$ for $30 \mathrm{~s}$ and $68{ }^{\circ} \mathrm{C}$ for $3 \mathrm{~min}$.

\section{Histology and immunohistochemistry}

Donated IS brains were routinely fixed in $10 \%$ neutral buffered formalin and trimmed in transverse and sagittal planes after longitudinal section in the midline. Amongst other levels, $0.3 \mathrm{~mm}$ serial sections of the cerebella and brainstem were embedded in paraffin after passing an ascending alcohol series and xylene immersion through an automatic tissue processor.

Our cerebellar staining panel included haematoxylineosin (HE), a modified Bielschowsky impregnation method (Erickson-Davis et al. 2010; Grimaldi and Manto 2012), and immunohistochemistry for synaptophysin (1:400, Syaptic Syystems GmbH, Goettingen, Germany), calbindin-D-28 K (1:1,000, Sigma, St. Louis, USA (Laure-Kamionowska and Maslinska 2009), microtubule associated protein-2 (MAP2; 1:1,000 (Resibois et al. 2007), inositol 1,4,5 tri-phosphate receptor-1 (ITPR1; 1:200; Linaris, Dossenheim, Germany (Miyata et al. 1999) and glial fibrillary acidic protein (GFAP; 1:500 Nobrega et al. 2013). Immunolabelling of Calbindin, MAP2 and ITPR1 employed microwave pretreatment in $\mathrm{pH} 6.0$ citrate buffer. Primary antibody binding was visualised using Impress ${ }^{\circledR}$ polymer technology (Linaris, Dossenheim, Germany) and a diaminobenzidine-based detection kit.

Assessment of the cerebellar morphology included lobulation and folia configuration, the subarachnoid space volume, cortical lamination pattern and cell density, as well as the morphology of foliary white matter, cerebellar roof nuclei, cerebellar peduncles, spinocerebellar tracts and the precerebellar pontine and olivary nuclei.

Purkinje cell features included their position and spatial distribution, the expression and subcellular distribution of differentiation markers, the dendritic tree configuration, the morphology of basket cell plexuses (Erickson-Davis et al. 2010) and the presence/absence of halo-like structures surrounding the PC (Yoshida et al. 2014).

\section{Results}

\section{Clinical investigations}

Neurological characteristics of SCAIS include a wide-based stance, spinocerebellar ataxia characterised by thoracic limb hypermetria (hyperextension), pelvic limb hyperflexion, truncal swaying, impaired balance, pendular nystagmus and absent menace response bilaterally. The remainder of the neurological examination was within normal limits. As the disease progressed intentional head tremor was observed and balance impairment deteriorated to the point that the dogs were unable to stand up and ambulate at approximately 1 year of age. Haematology, serum biochemistry and urinalysis were unremarkable in all affected dogs. MRI of the brain of two affected 8-month-old littermates (one male and one female) showed a subjective mild increase in sulcal widths and exaggerated definition of the folia in the rostral lobe of the cerebellum, suggesting grey matter loss. In addition, a cerebellar vermian defect was noted in the ventral portion of the cerebellum. However, these MRI changes were observed also on MRI of a clinically normal female littermate unaffected by the ITPRI mutation, and therefore their clinical significance remains dubious. MRI was repeated in the affected female dog 5.5 weeks after the first one; however, no changes were observed despite significant clinical deterioration. MRI of the cervical spine revealed no abnormalities in the two IS undergoing cervical MRI. The clinically normal individual subjected to MRI was followed up for several years by a veterinary neurologist and did not develop any clinical signs of spinocerebellar ataxia. The MRI results therefore cannot be seen as diagnostic of SCAIS and may just have shown mild anatomic variants of no clinical significance. Cisternal cerebrospinal fluid (CSF) analysis was normal (in four tested dogs) and CSF PCRs for canine distemper virus, Toxoplasma gondii and Neospora caninum were negative (in two tested dogs). Brain stem auditory evoked response testing was normal (in four tested dogs).

\section{Genetics investigations}

Using a genome-wide homozygosity mapping approach with six Italian Spinoni diagnosed with SCAIS and six controls IS, we identified two microsatellite markers, C20.374 and Ren124F16, that showed a pattern suggestive of linkage to chromosome 20 of the canine genome. All SCAIS cases were homozygous and all controls heterozygous for a two marker haplotype (Table 1).

Linkage analysis across an extended pedigree containing 13 cases and 47 controls, including 16 obligate carriers, was performed to confirm the association of SCA to chromosome 20. Data from 22 microsatellite markers across chromosome 20 were analysed using the MLINK software package, with marker $\mathrm{C} 20.374$ achieving a maximal logarithm of odds (LOD) score of 4.41 at $\theta 0$ (Fig. 1), confirming linkage.

Upon confirmation of linkage to chromosome 20 fine mapping was performed using additional markers to define 
Table 1 Homozygosity mapping results suggesting linkage of SCAIS to CFA20. Markers C20.374 and Ren124F16 gave results suggestive of linkage to SCAIS

(A) All cases are homozygous for the same shared two marker haplotype. (B) Genotypes for control individuals

\begin{tabular}{|c|c|c|c|c|c|c|c|}
\hline \multicolumn{8}{|l|}{ (A) } \\
\hline \multirow[t]{2}{*}{ Marker } & \multirow{2}{*}{$\begin{array}{l}\text { Position } \\
\text { (Chromosome: Mb) }\end{array}$} & \multicolumn{6}{|c|}{ Case ID number } \\
\hline & & 5357 & 5397 & 5404 & 6422 & 6477 & 6685 \\
\hline C20.374 & $20: 15.60$ & 191/191 & $191 / 191$ & 191/191 & $191 / 191$ & $191 / 191$ & $191 / 191$ \\
\hline Ren124F16 & $20: 21.93$ & $233 / 233$ & $233 / 233$ & $233 / 233$ & $233 / 233$ & $233 / 233$ & $233 / 233$ \\
\hline \multicolumn{8}{|l|}{ (B) } \\
\hline \multirow[t]{2}{*}{ Markers } & Position & \multicolumn{6}{|c|}{ Control ID number } \\
\hline & (Chromosome: Mb) & 5297 & 5298 & 5405 & 5407 & 5436 & 6478 \\
\hline C20.374 & $20: 15.60$ & $187 / 191$ & $191 / 191$ & $182 / 191$ & $182 / 191$ & $191 / 191$ & $191 / 191$ \\
\hline Ren124F16 & $20: 21.93$ & $233 / 238$ & $238 / 240$ & $233 / 233$ & $233 / 233$ & $233 / 238$ & $233 / 238$ \\
\hline
\end{tabular}

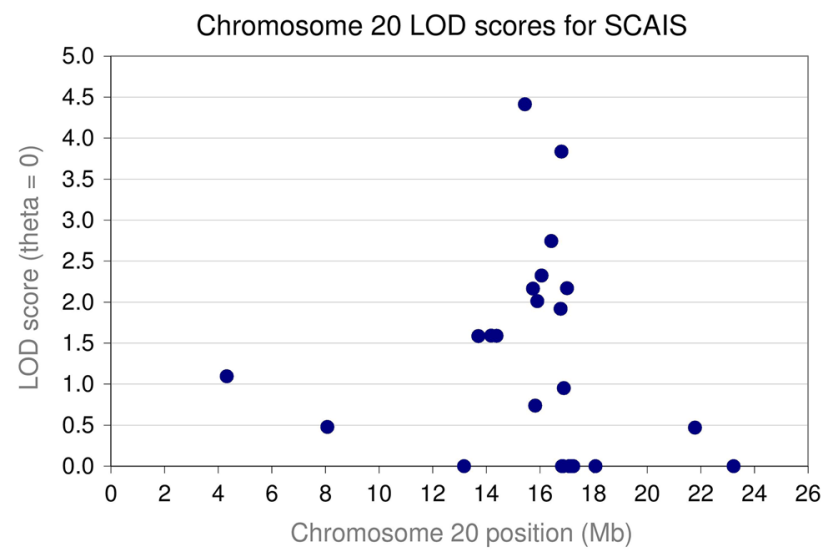

Fig. 1 Plot of chromosome 20 LOD scores associated with CFA20 markers when genotyped across an extended IS pedigree consisting of 13 SCA cases and 47 controls. The maximal LOD score was 4.41 at $15.60 \mathrm{Mb}$

the disease-associated haplotype. The 13 SCAIS cases were genotyped across a total of 40 microsatellite and 8 SNP markers, defining the disease associated

interval as chr20:15,601,140-17,116,778 based on the genomic coordinates of the CanFam2 assembly (Supplementary File 1). The disease-associated interval contained 5 genes: BHLHE40, ITPRI, SUMF1, SETMAR and LRRN1. All five genes were exon resequenced, although no polymorphisms were identified which segregated consistently with disease status.

A massively parallel sequencing experiment was subsequently undertaken, using a probe-based target enrichment methodology to capture the entire disease-associated region of two SCAIS cases and three controls. Controls with haplotypes similar to the disease-associated haplotype were selected to reduce the number of potentially causal variants. A $20 \mathrm{~Gb}$ dataset of $51 \mathrm{bp}$ paired-end reads was produced. Across the $1.52 \mathrm{Mb}$ disease-associated interval a total of 3,871 SNPs and 622 INDELs were identified, although only eight of these variants segregated in accordance with disease status. All eight segregating variants could be ruled out by genotyping additional control individuals. On visual scanning of the sequence read alignments, a $30 \mathrm{bp}$ region showing variable coverage between cases and controls was identified (Fig. 2). In this region, read depth dropped to zero for cases but remained in all three controls, although the profile of the coverage histogram is comparable for all individuals. Most of the unsequenced region, which was located in intron 35 of ITPRI, consisted of a poly(T) tract $5^{\prime}$ of a SINE element and $3^{\prime}$ of a short trinucleotide repeat sequence $\operatorname{TTC}_{(8)}$. Just upstream of the region many reads were present which had unaligned mates (singleton reads). Further investigation revealed the sequence of the unaligned mates to be $\mathrm{GAA}_{(17)}$ suggesting expansion of the trinucleotide repeat sequence.

Long range PCR was used to confirm expansion of the region in cases. Amplification was only achievable using highly intact genomic DNA, meaning no results could be obtained for 6 of the 13 cases for which DNA was obtained from FFPE brain tissue. The seven remaining cases all had two expanded alleles, although none of the cases had two alleles of identical length even though the cases were all homozygous for an identical disease-associated haplotype which is indicative of a founding expansion event. Allele size ranges are demonstrated in Fig. 3. Expanded alleles were estimated to range from 318 to 651 GAA repeats in length (Supplementary File 2). All 15 obligate carriers in our sample collection were heterozygous for the repeat expansion, with non-expanded alleles ranging from approximately 7-22 subunits. Both intergeneration expansion and reduction in repeat copy number were observed. The expansion was confirmed as a pure GAA repeat expansion by Sanger sequencing of a gel extracted case allele amplicon (Fig. 4). 
Fig. 2 View in IGV of reads aligned to intron 35 of canine ITPRI for one case and one control individual. The green triangle indicates the position where coverage is only achieved for the control individual but not the SCAIS case. Reads (grey bars) with a red perimeter indicate singleton reads. Unaligned mates of singleton reads are of the sequence $\mathrm{GAA}_{(17)}$, suggesting repeat expansion (reference sequence $\left.\mathrm{GAA}_{(8)}\right)$ (Color figure online)

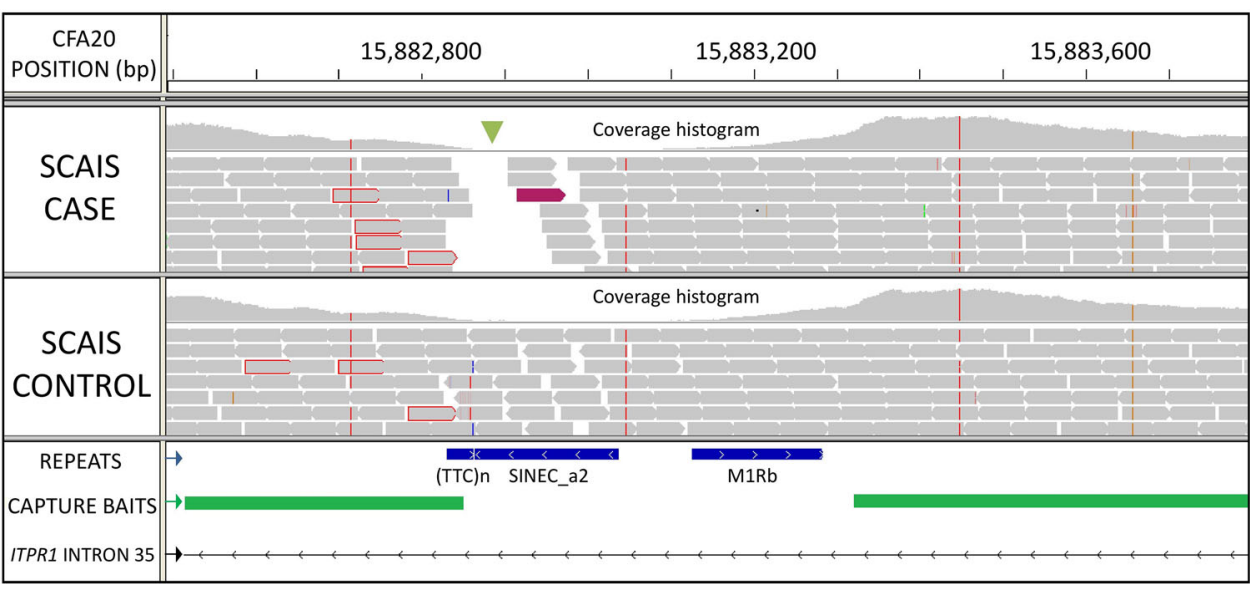

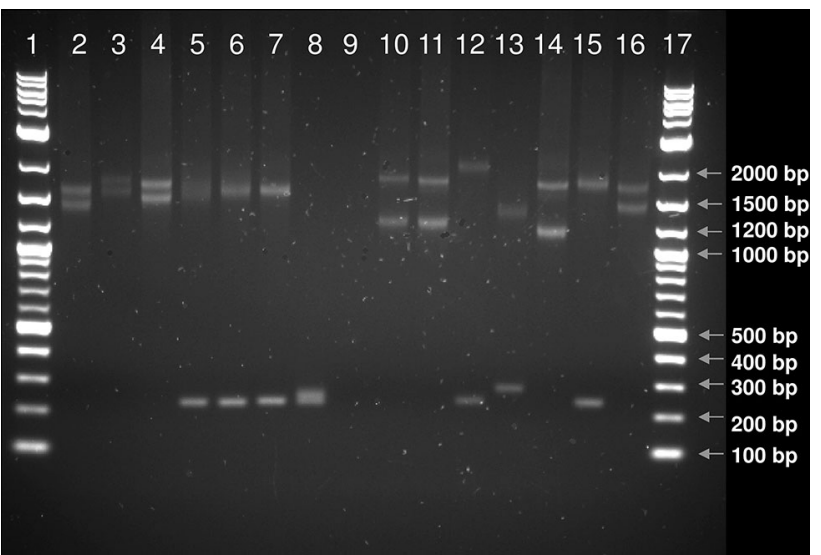

Fig. 3 PCR analysis of the GAA repeat polymorphism. The expected wild-type allele size of the amplicon crossing the ITPR1 GAA triplet repeat expansion $238 \mathrm{bp}$. PCR products from cases are in lane 2, 3, 4, $10,11,14$ and 16, from obligate carriers in lanes 5, 6, 7, 12, 1314 and 15 and from a homozygous wild-type individual in lane 8. Lane 9 contains a no-template control. All cases have two expanded alleles of varying lengths. Results suggest expanded alleles ranging from $\mathrm{GAA}_{300}-\mathrm{GAA}_{650}$

\section{Histology and ITPR1 expression}

The overall size and volume ratios of ITPR1 mutant IS cerebella as well as the lobule and folia formation, the diameters of the fissures and sulci and the area of the subarachnoid space were within a normal range. Cerebellocortical layers were sharply delineated, the Purkinje cells (PC) were correctly placed and the granule cell layer presented with normal density and glomerula formation. Mild variations of the PC density (Fig. 5a) corresponded to the sublobular segmental pattern seen in age-matched control IS. In affected IS, the molecular layer exhibited some focal stellate cell hypercellularity (Fig. 5a). Dendritic and somatic changes of PC morphology were not evident. Fluorojade C accumulation was not seen in cerebellar neurons. On HE,
peri-PC basket cell plexuses (BCP) appeared prominent (Fig. 5b). They stained physiologically with synaptophysin (Fig. 5c). Synaptophysin-positive halo-like structures, such as seen in human SCA-31 (Yoshida et al. 2014) were excluded. Upon Bielschowskýs stain, BCP were sorted to type 0 to 2 at variable frequencies (Erickson-Davis et al. 2010) as they were in age-matched control dogs. Very occasionally $(<1 \mathrm{BCP}$ per lobule), empty baskets were detected (Fig. 5e). Even in morphologically inconspicuous PC, calbindin-D-28 k expression was inconstant and failed to highlight the dendritic morphology and orientation due to weak immunopositivity (Fig. 6b). Only a minority of PC (10-15\%) expressed ITPR1 strongly, with additional discrepancies regarding perikaryal versus dendritic staining. In control dogs, $100 \%$ of Purkinje cells show strong ITPR 1 expression throughout all lobules. Areas of ITPR1 expression in cases, however, allowed for identification of a distortion of the of monoplanar orientation of the dendritic trees. Instead of the two-dimensional arborisation in sagittal plane, the dendrites, now birch-broom-like, extended into the molecular layer towards the pial membrane. Thereby, secondary and tertiary dendrites and spiny branchlets left the stem at a moderately steep angle (Fig. 6d). Both reduced ITPR1 expression and the defective planar orientation of immunopositive cells involved all lobules and functional subfields of the affected cerebella with a mild emphasis on the spinocerebellar parts of the vermis. This notably colocalised with a moderately increased GFAP immunopositivity of Bergmann glia processes in the outer molecular layer. Bergmanńs astrocytosis was not evident on HE nor GFAP stained slides. MAP2 immunohistochemstry revealed a normal functional integration of stellate cells, basket cells, Golgi cells and granular cells. The overall histological appearance of the large afferent/efferent fibre tracks, cerebellar roof and feedback nuclei was normal. Only the density of ITPR1 positive presynapses appeared reduced in the cerebellar roof nuclei and vestibular nuclei. 


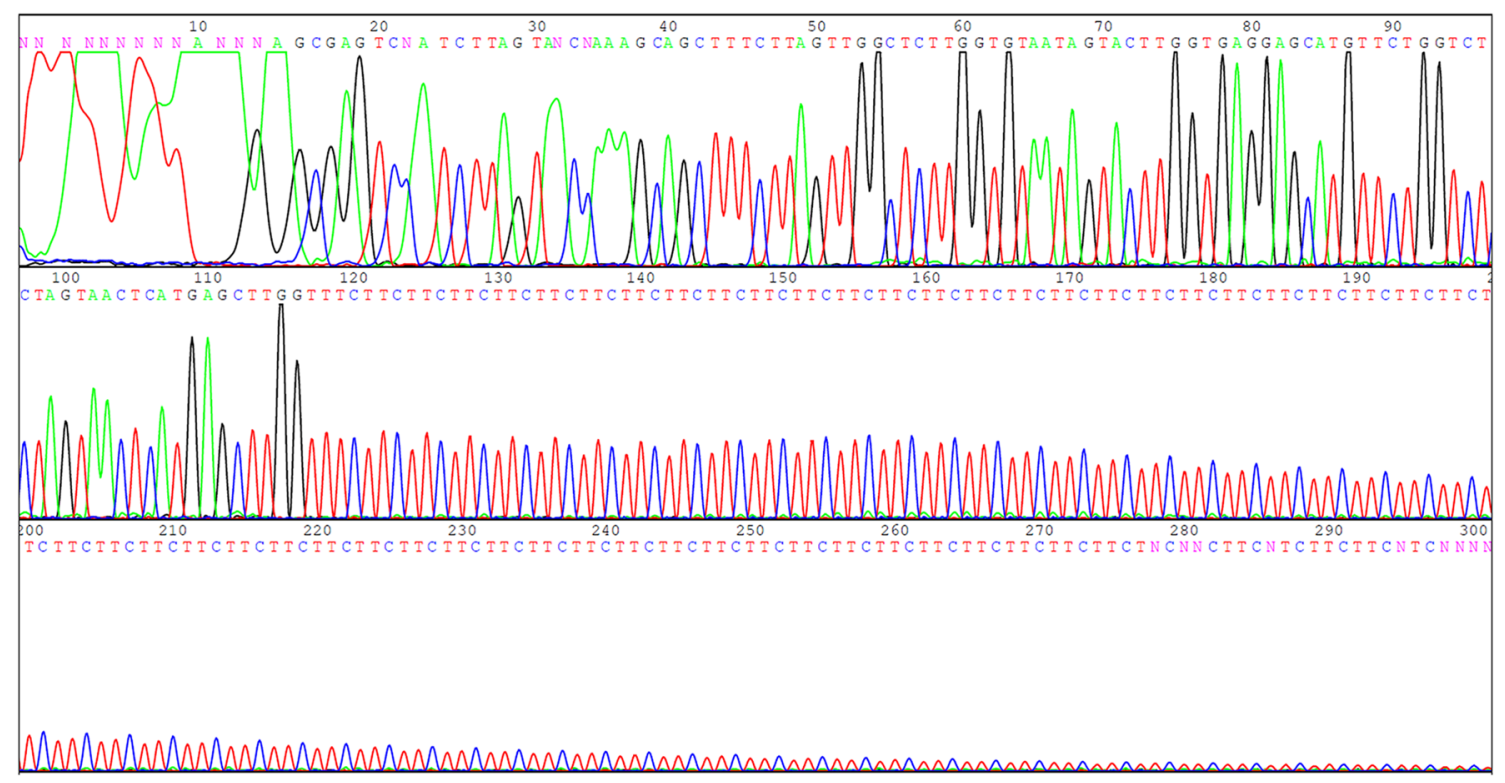

Fig. 4 Sanger sequencing trace of the GAA (CTT) repeat expansion. Sanger sequencing of an expanded amplicon produced from a SCA case confirmed a pure GAA repeat expansion, although a complete trace spanning the entire length of the GAA repeat could not be produced

\section{Discussion}

We have identified a GAA repeat expansion in intron 35 ITPRI which is strongly associated with autosomal recessive SCAIS. Cases in the study had expanded alleles in the range of approximately $\mathrm{GAA}_{(300)}$ to $\mathrm{GAA}_{(650)}$ in comparison to a wild-type range of $\mathrm{GAA}_{(7)}$ to $\mathrm{GAA}_{(22)}$. All cases shared a common disease-associated haplotype, but had two expanded repeat alleles of varying length, implying that repeat length is unstable and generational expansion and contraction was observed.

The GAA expansion discovered in the IS is similar to the repeat expansion causing Friedreich ataxia in humans (Campuzano et al. 1996). Both expansions stem from a short GAA repeat on the edge of a SINE element, and result in autosomal recessive ataxic conditions. The intronic GAA repeat expansion causing Friedreich ataxia is located in intron one of the frataxin $(F X N)$ gene, and results in reduced expression of the FXN transcript, potentially due to the formation of a triplex DNA structure and "sticky DNA" (Sakamoto et al. 1999).The mechanism by which reduced frataxin, a mitochondrial protein involved in ironsulphur cluster regulation, causes neurodegeneration is debated, but recent research support a neuroinflammatory mechanism (Adinolfi et al. 2009; Hayashi et al. 2014). Triplet repeat copy number in Friedreich ataxia patients has been reported to range between 66 and 1,700 subunits, thus the GAA repeat expansion observed in canine ITPRI falls within this range (Durr et al. 1996; Epplen et al. 1997). Like the repeat expansion event in the IS there is evidence that Friedrich ataxia expansion alleles have a common founder (Cossee et al. 1997). Furthermore, large normal alleles also exist, which have arisen from a single founding chromosome. These large normal alleles in frataxin represent a pre-mutation reservoir and catastrophic single generation normal range to pathogenic repeat range changes have been observed (Cossee et al. 1997). This evidence suggests that although this form of cerebellar ataxia has been eradicated in the IS through a selective breeding programme, expansion of a large normal allele is possible, which could potentially cause a resurgence of the disease within the breed population. It also raises the possibility that a similar repeat expansion could be the cause of ataxia in other dog breeds.

The gene ITPRI encodes inositol 1,4,5-trisphosphate receptor type 1, which is an intracellular inositol tri-phosphate (IP3)-gated calcium channel, which regulates the release of calcium from the cytoplasm from intracellular stores. Signalling through IP3 and calcium release is involved in a number of cellular processes including cell division, synaptic transmission, gene expression and apoptosis (Foskett 2010). Heterozygous deletion of the ITPRI gene has been shown to cause SCA15 (also designated SCA16) in humans (Huang et al. 2012). SCA15 is an autosomal dominant pure cerebellar ataxia with a reported prevalence of $1.8 \%$ of patients affected by SCA (Marelli et al. 2011) and an onset age ranging from 10 to 66 years (Miyoshi et al. 2001; Storey et al. 2001). Using EBV immortalised lymphocytes, ITPR1 protein levels were shown to be considerably lower in cell lines derived from affected individuals, and haploinsufficiency had been suggested as the cause of the disease. Mice lacking ITPRI displays a 

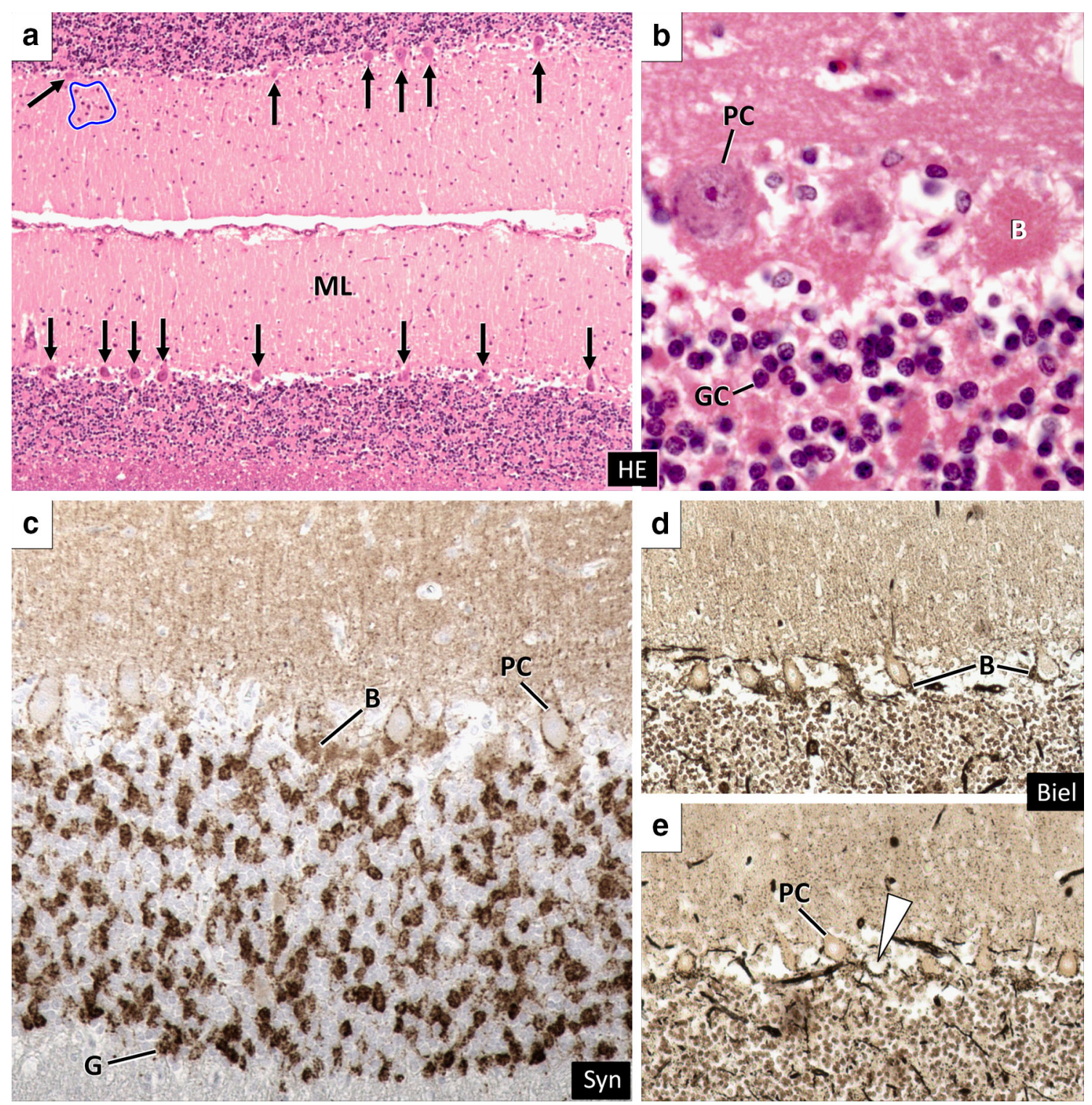

Fig. 5 Cerebellar cortex in ITPR1 mutant IS (a-c, e) versus controls (d). Cortical layering and Purkinje cell (PC) density (a black arrows) are within a normal range. The molecular layer (ML) shows occasional foci of mildly increased stellate cell density (a blue line). Dendritic and somatic PC changes are not evident on HE slides (a, b). Instead,

severe phenotype, although most die in utero. Surviving mice show normal behaviour at birth with signs of ataxia apparent at day nine. Tonic or tonic/clonic seizures develop at day 20-23 and mice die by the weaning period (Matsumoto et al. 1996). A similar but less severe phenotype is displayed by the opt mouse, which has an in-frame deletion of exons 43 and 44 of ITPRI (Street et al. 1997). Extracerebellar signs may be due to lesions in other ITPR 1 rich brain regions such as the hippocampal CA1 segment, basal nuclei and thalamus in knock-out mice. Human SCA15 and other genetic ataxias that imply perturbation of the baskets $(B)$ appear slightly prominent (b) on HE. The basket cell plexus morphology, on the other hand, is normal on synaptophysin (Syn c) immunohistochemistry and on Bielschowskýs stain (Biel e). Very rarely, empty baskets are seen in the vermis (e arrowhead). GC granule cell, $G$ synaptic glomerulus (Color figure online)

ITPR1-signalling pathways, such as due to CACNA1A, $P K C G$ and $C A C N 4 B$ mutation, in contrast, presents mostly as monosystem disease with pure rather than complex cerebellar dysfunction. Also for SCAIS, extracerebellar signs have not been identified. The ataxia, moreover, is less severe than in the mouse, due to incomplete loss of ITPRI expression.

As in human SCA15, ITPRl expression is partially impaired in homozygous IS, explaining the attenuated phenotype if compared to the mouse. Disruption of ITPR1dependent signalling is thought to affect long-term 

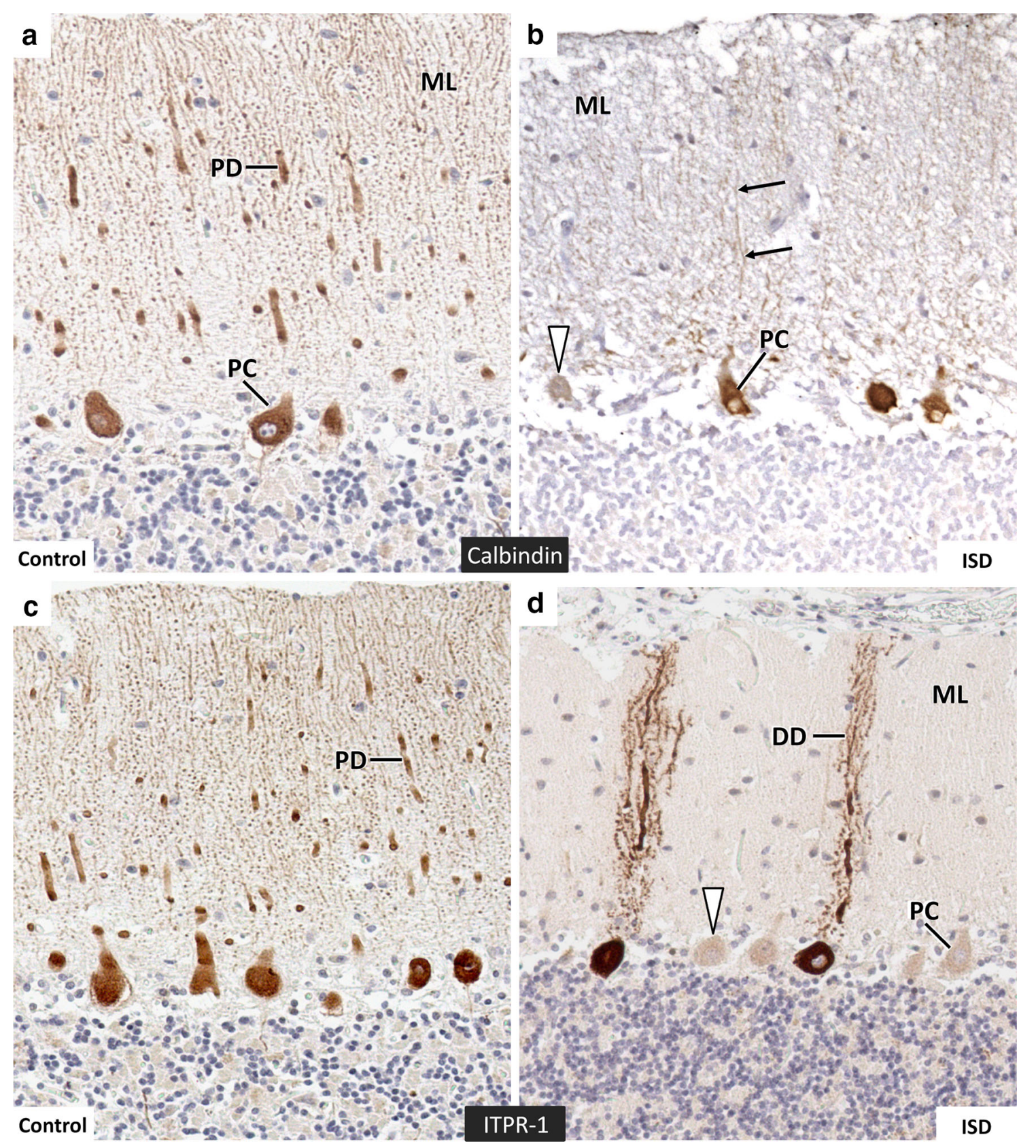

Fig. 6 Dendritic morphology and expression of calbindin-D-28k and ITPR1 in SCA-affected IS (b, d) as compared to controls (a, c). On calbindin-D-28 k immunohistochemistry (b), ITPR1 mutant IS shows variable and inconstant antigen expression with scattered immunonegative PC somata (arrowhead) as well as with poorly stained PC

depression and the heterosynaptic plasticity in Purkinje cells with severe effects on motor coordination and learning as well as on maturation of PC dendrite morphology and synaptogenesis (Schorge et al. 2010). Moreover, the histological evidence of degenerative features and reactive astrogliosis in the IS brains offers the possibility of phenotypic worsening by premature drop out of functional PC. Current reports on ITPR1 mouse mutants failed to dendrites (black arrows). A majority of about $90 \%$ of PC stains negative or weakly positive for ITPR1 (d arrowhead). Stronger ITPR1 expression highlights the loss of planar orientation and dysmorphology of the PC dendritic tree (d DD). PD planar dendritic tree, $D D$ dysmorphic dendritic tree, $M L$ molecular layer

document changes other than the reduction of ITPR1 immunoreactivity amongst PC (van de Leemput et al. 2007). The authors do not comment on PC cytomorphology or cerebellocortical architecture. In the same vein, there are no data available on cerebellar histomorphology in SCA15 patients. Hence, ITPRI mutant IS may help to increase our understanding of the morphological substrate underlying this type of hereditary ataxia. 
Unlike human SCA15 cases, however, ataxia has not been observed in heterozygous dogs, perhaps because more ITPRI transcript is produced than in haploinsufficient human cases. Alterative explanations include insufficient canine longevity to allow disease progression or failure of owners to notice phenotypic changes which may be more subtle in quadrupeds.

Identification of an intronic causal mutation highlights the limitations of using popular enrichment techniques such as exome capture for targeted resequencing as a means of mutation identification. Although the intronic GAA repeat expansion was identified using target enrichment of the disease-associated interval, mutation identification was actually facilitated by repeat masking preventing capture probes (baits) being designed across the repeat expansion location. In controls sufficient flanking sequence was captured by baits to result in sequence coverage all the way through the repetitive region. For cases, however, because the repeat expansion increased the size of the masked repetitive region, no DNA fragments were captured that were of sufficient size to span across the GAA repeat. This resulted in a region of zero coverage in cases $3^{\prime}$ of the GAA repeat sequence, as no probes $3^{\prime}$ of the GAA repeat were located close enough to capture this region. The gap in coverage in cases, but not controls provided a discernable difference that could be followed up allowing mutation identification. We speculate that the repeat expansion may also have been difficult to identify using a whole-genome sequencing approach due to the intronic positioning and because complete coverage would be achieved for both cases and controls, although an increase in singleton reads would still be observed across the region for IS cases. The repetitive nature and intronic location of the associated variant made identification challenging, with the study taking several years to complete, hence the range of classic and more modern techniques used.

To conclude, we have identified a repeat expansion in intron 35 of ITPRI leading to impaired protein expression in PC which is strongly associated with SCAIS. No repeat expansions in ITPRI have been associated with cerebellar ataxia previously and this is the first reported naturally occurring pathogenic intronic repeat expansion in a nonhuman species.

Acknowledgments We would like to thank the many dog owners that have contributed to this study. We thank the High-Throughput Genomics Group at the Wellcome Trust Centre for Human Genetics (funded by Wellcome Trust Grant reference 090532/Z/09/Z and MRC Hub Grant G0900747 91070) for the generation of the sequencing data. This study was funded by the Italian Spinone Club of Great Britain, the Kennel Club Charitable Trust, and the Animal Health Trust.
Ethical standards The authors declare that the experiments comply with the current laws of the country in which they were performed.

Conflict of interest The authors declare that they have no conflict of interest.

Open Access This article is distributed under the terms of the Creative Commons Attribution License which permits any use, distribution, and reproduction in any medium, provided the original author(s) and the source are credited.

\section{References}

Adinolfi S, Iannuzzi C, Prischi F, Pastore C, Iametti S, Martin SR, Bonomi F, Pastore A (2009) Bacterial frataxin CyaY is the gatekeeper of iron-sulfur cluster formation catalyzed by IscS. Nat Struct Mol Biol 16:390-396

Campuzano V, Montermini L, Molto MD, Pianese L, Cossee M, Cavalcanti F, Monros E, Rodius F, Duclos F, Monticelli A, Zara F, Canizares J, Koutnikova H, Bidichandani SI, Gellera C, Brice A, Trouillas P, De Michele G, Filla A, De Frutos R, Palau F, Patel PI, Di Donato S, Mandel JL, Cocozza S, Koenig M, Pandolfo M (1996) Friedreich's ataxia: autosomal recessive disease caused by an intronic GAA triplet repeat expansion. Science 271:1423-1427

Cossee M, Schmitt M, Campuzano V, Reutenauer L, Moutou C, Mandel JL, Koenig M (1997) Evolution of the Friedreich's ataxia trinucleotide repeat expansion: founder effect and premutations. Proc Natl Acad Sci U S A 94:7452-7457

Durr A, Cossee M, Agid Y, Campuzano V, Mignard C, Penet C, Mandel JL, Brice A, Koenig M (1996) Clinical and genetic abnormalities in patients with Friedreich's ataxia. N Engl J Med 335:1169-1175

Epplen C, Epplen JT, Frank G, Miterski B, Santos EJ, Schols L (1997) Differential stability of the (GAA)n tract in the Friedreich ataxia (STM7) gene. Hum Genet 99:834-836

Erickson-Davis CR, Faust PL, Vonsattel JP, Gupta S, Honig LS, Louis ED (2010) "Hairy baskets" associated with degenerative Purkinje cell changes in essential tremor. J Neuropathol Exp Neurol 69:262-271

Forman OP, De Risio L, Stewart J, Mellersh CS, Beltran E (2012) Genome-wide mRNA sequencing of a single canine cerebellar cortical degeneration case leads to the identification of a disease associated SPTBN2 mutation. BMC Genet 13:55

Foskett JK (2010) Inositol trisphosphate receptor $\mathrm{Ca} 2+$ release channels in neurological diseases. Pflugers Arch 460:481-494

Grimaldi G, Manto M (2012) Mechanisms and emerging therapies in tremor disorders. Springer, New York

Hayashi G, Shen Y, Pedersen TL, Newman JW, Pook M, Cortopassi G (2014) Frataxin deficiency increases cyclooxygenase 2 and prostaglandins in cell and animal models of Friedreich's ataxia. Hum Mol Genet http://www.ncbi.nlm.nih.gov/pubmed/25104852

Higgins JJ, Nee LE, Vasconcelos O, Ide SE, Lavedan C, Goldfarb LG, Polymeropoulos MH (1996) Mutations in American families with spinocerebellar ataxia (SCA) type 3: SCA3 is allelic to Machado-Joseph disease. Neurology 46:208-213

Holmes SE, O'Hearn EE, McInnis MG, Gorelick-Feldman DA, Kleiderlein JJ, Callahan C, Kwak NG, Ingersoll-Ashworth RG, Sherr M, Sumner AJ, Sharp AH, Ananth U, Seltzer WK, Boss MA, Vieria-Saecker AM, Epplen JT, Riess O, Ross CA, Margolis RL (1999) Expansion of a novel CAG trinucleotide 
repeat in the $5^{\prime}$ region of PPP2R2B is associated with SCA12. Nat Genet 23:391-392

Huang L, Warman J, Carter MT, Friend KL, Dudding TE, Schwartzentruber J, Zou R, Schofield PW, Douglas S, Bulman DE, Boycott KM (2012) Missense mutations in ITPR1 cause autosomal dominant congenital nonprogressive spinocerebellar ataxia. Orphanet J Rare Dis 7:67

Koide R, Kobayashi S, Shimohata T, Ikeuchi T, Maruyama M, Saito M, Yamada M, Takahashi H, Tsuji S (1999) A neurological disease caused by an expanded CAG trinucleotide repeat in the TATA-binding protein gene: a new polyglutamine disease? Hum Mol Genet 8:2047-2053

Koob MD, Moseley ML, Schut LJ, Benzow KA, Bird TD, Day JW, Ranum LP (1999) An untranslated CTG expansion causes a novel form of spinocerebellar ataxia (SCA8). Nat Genet 21:379-384

Laure-Kamionowska M, Maslinska D (2009) Calbindin positive Purkinje cells in the pathology of human cerebellum occurring at the time of its development. Folia Neuropathol 47:300-305

Li H, Durbin R (2009) Fast and accurate short read alignment with Burrows-Wheeler transform. Bioinformatics 25:1754-1760

Lindblad K, Savontaus ML, Stevanin G, Holmberg M, Digre K, Zander C, Ehrsson H, David G, Benomar A, Nikoskelainen E, Trottier Y, Holmgren G, Ptacek LJ, Anttinen A, Brice A, Schalling M (1996) An expanded CAG repeat sequence in spinocerebellar ataxia type 7. Genome Res 6:965-971

Marelli C, van de Leemput J, Johnson JO, Tison F, Thauvin-Robinet C, Picard F, Tranchant C, Hernandez DG, Huttin B, Boulliat J, Sangla I, Marescaux C, Brique S, Dollfus H, Arepalli S, Benatru I, Ollagnon E, Forlani S, Hardy J, Stevanin G, Durr A, Singleton A, Brice A (2011) SCA15 due to large ITPR1 deletions in a cohort of 333 white families with dominant ataxia. Arch Neurol 68:637-643

Matsumoto M, Nakagawa T, Inoue T, Nagata E, Tanaka K, Takano H, Minowa O, Kuno J, Sakakibara S, Yamada M, Yoneshima H, Miyawaki A, Fukuuchi Y, Furuichi T, Okano H, Mikoshiba K, Noda T (1996) Ataxia and epileptic seizures in mice lacking type 1 inositol 1,4,5-trisphosphate receptor. Nature 379:168-171

Matsuura T, Yamagata T, Burgess DL, Rasmussen A, Grewal RP, Watase K, Khajavi M, McCall AE, Davis CF, Zu L, Achari M, Pulst SM, Alonso E, Noebels JL, Nelson DL, Zoghbi HY, Ashizawa T (2000) Large expansion of the ATTCT pentanucleotide repeat in spinocerebellar ataxia type 10 . Nat Genet 26:191-194

McKenna A, Hanna M, Banks E, Sivachenko A, Cibulskis K, Kernytsky A, Garimella K, Altshuler D, Gabriel S, Daly M, DePristo MA (2010) The genome analysis toolkit: a MapReduce framework for analyzing next-generation DNA sequencing data. Genome Res 20:1297-1303

Miyata M, Miyata H, Mikoshiba K, Ohama E (1999) Development of Purkinje cells in humans: an immunohistochemical study using a monoclonal antibody against the inositol 1,4,5-triphosphate type 1 receptor (IP3R1). Acta Neuropathol 98:226-232

Miyoshi Y, Yamada T, Tanimura M, Taniwaki T, Arakawa K, Ohyagi Y, Furuya H, Yamamoto K, Sakai K, Sasazuki T, Kira J (2001) A novel autosomal dominant spinocerebellar ataxia (SCA16) linked to chromosome 8q22.1-24.1. Neurology 57:96-100
Nobrega C, Nascimento-Ferreira I, Onofre I, Albuquerque D, Hirai H, Deglon N, de Almeida LP (2013) Silencing mutant ataxin-3 rescues motor deficits and neuropathology in Machado-Joseph disease transgenic mice. PLoS ONE 8:e52396

Orr HT, Chung MY, Banfi S, Kwiatkowski TJ Jr, Servadio A, Beaudet AL, McCall AE, Duvick LA, Ranum LP, Zoghbi HY (1993) Expansion of an unstable trinucleotide CAG repeat in spinocerebellar ataxia type 1 . Nat Genet 4:221-226

Resibois A, Coppens A, Poncelet L (2007) Naturally occurring parvovirus-associated feline hypogranular cerebellar hypoplasia- A comparison to experimentally-induced lesions using immunohistology. Vet Pathol 44:831-841

Riess O, Schols L, Bottger H, Nolte D, Vieira-Saecker AM, Schimming C, Kreuz F, Macek M Jr, Krebsova A, Macek MS, Klockgether T, Zuhlke C, Laccone FA (1997) SCA6 is caused by moderate CAG expansion in the alpha1A-voltage-dependent calcium channel gene. Hum Mol Genet 6:1289-1293

Sakamoto N, Chastain PD, Parniewski P, Ohshima K, Pandolfo M, Griffith JD, Wells RD (1999) Sticky DNA: self-association properties of long GAA.TTC repeats in R.R.Y triplex structures from Friedreich's ataxia. Mol Cell 3:465-475

Sanpei K, Takano H, Igarashi S, Sato T, Oyake M, Sasaki H, Wakisaka A, Tashiro K, Ishida Y, Ikeuchi T, Koide R, Saito M, Sato A, Tanaka T, Hanyu S, Takiyama Y, Nishizawa M, Shimizu N, Nomura Y, Segawa M, Iwabuchi K, Eguchi I, Tanaka H, Takahashi H, Tsuji S (1996) Identification of the spinocerebellar ataxia type 2 gene using a direct identification of repeat expansion and cloning technique, DIRECT. Nat Genet 14:277-284

Schorge S, van de Leemput J, Singleton A, Houlden H, Hardy J (2010) Human ataxias: a genetic dissection of inositol triphosphate receptor (ITPR1)-dependent signaling. Trends Neurosci 33:211-219

Storey E, Gardner RJ, Knight MA, Kennerson ML, Tuck RR, Forrest SM, Nicholson GA (2001) A new autosomal dominant pure cerebellar ataxia. Neurology 57:1913-1915

Street VA, Bosma MM, Demas VP, Regan MR, Lin DD, Robinson LC, Agnew WS, Tempel BL (1997) The type 1 inositol 1,4,5trisphosphate receptor gene is altered in the opisthotonos mouse. J Neurosci 17:635-645

Thorvaldsdottir H, Robinson JT, Mesirov JP (2013) Integrative genomics viewer (IGV): high-performance genomics data visualization and exploration. Brief Bioinform 14:178-192

van de Leemput J, Chandran J, Knight MA, Holtzclaw LA, Scholz S, Cookson MR, Houlden H, Gwinn-Hardy K, Fung HC, Lin X, Hernandez D, Simon-Sanchez J, Wood NW, Giunti P, Rafferty I, Hardy J, Storey E, Gardner RJ, Forrest SM, Fisher EM, Russell JT, Cai H, Singleton AB (2007) Deletion at ITPR1 underlies ataxia in mice and spinocerebellar ataxia 15 in humans. PLoS Genet 3:e108

Wheeler S, Rusbridge C (1996) Neurological syndrome in Italian spinones. Vet Rec 138:216

Yoshida K, Asakawa M, Suzuki-Kouyama E, Tabata K, Shintaku M, Ikeda S, Oyanagi K (2014) Distinctive features of degenerating Purkinje cells in spinocerebellar ataxia type 31 . Neuropathology 34:261-267 